\title{
ASPECTOS FLORÍSTICOS DA VEGETAÇÃO DE RESTINGA NO LITORAL DE PERNAMBUCO ${ }^{1}$
}

\author{
Ana Cláudia Sacramento ${ }^{2}$, Carmen Sílvia Zickel ${ }^{3}$ e Eduardo Bezerra de Almeida Jr. ${ }^{4}$
}

\begin{abstract}
RESUMO - As zonas costeiras acolhem quadros de alta riqueza e relevância ecológica que as qualificam como importante ecossistema para conservação. O objetivo deste estudo foi listar as espécies que compõem a restinga da praia do Paiva e descrever a sua fisionomia. A área de restinga compreende 147 ha, situa-se no Município do Cabo de Santo Agostinho, PE, sob as coordenadas $08^{\circ} 07^{\prime} 30^{\prime \prime} \mathrm{S}$ e $35^{\circ} 00^{\prime} 55^{\prime \prime} \mathrm{W}$. As coletas florísticas foram realizadas durante 28 meses, em todos os estratos. O solo foi classificado como Neossolo Quartzarênico; foram determinadas duas fisionomias: a floresta não-inundável e o fruticeto aberto não-inundável. Foram listadas 124 espécies, distribuídas em 103 gêneros e 55 famílias. As famílias com maior número de espécies foram Myrtaceae, com 11 espécies, Cyperaceae (10), Fabaceae (8), Euphorbiaceae (7), Rubiaceae (6) e Asteraceae (5). As espécies Anacardium occidentale, Tapirira guianensis, Chamaecrista ramosa, Protium heptaphyllum, Byrsonima sericea, Myrcia rotundifolia e Marlierea schotti são encontradas na maioria das restingas do Nordeste. No entanto, devido à incipiência dos estudos na Região não foram verificadas espécies endêmicas.
\end{abstract}

Palavras-chave: Restinga, flora e fisionomia.

\section{FLORISTIC ASPECTS OF RESTINGA VEGETATION IN THE COAST OF PERNAMBUCO - BRAZIL}

\begin{abstract}
The high biodiversity and richness of the coastal zone make it an important ecosystem to be preserved. The aim of this study is to list the species composition of the restinga area (147 ha.) of Paiva

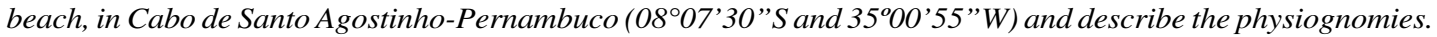
Floristic survey was carried out for 28 months for all strata. The soil was classified as Quartzarenic Neosoil and two physiognomies were determined: unflooded forest and unflooded open shrubby vegetation. One hundredtwenty-four species were recorded, distributed in 103 genera and 55 families. The families with the highest number of species were: Myrtaceae (11), Cyperaceae (10), Fabaceae (8), Euphorbiaceae (7), Rubiaceae (6) and Asteraceae (5). Anacardium occidentale, Byrsonima sericea, Chamaecrista ramosa, Marlierea schotti, Myrcia rotundifolia, Protium heptaphyllum and Tapirira guianensis are generally found in the majority of the sandy coastal plain in northeastern Brazil. However, due to insufficient studies, endemic species were not detected.
\end{abstract}

Keywords: Restinga vegetation, floristic composition and physiognomies.

\footnotetext{
${ }^{1}$ Recebido em 26.06.2006 e aceito para publicação em 21.11.2007.

${ }^{2}$ CPRH, São Lourenço da Mata. E-mail: <cszickel@ hotmail.com>.

${ }^{3}$ Departamento de Biologia -Botânica da Universidade Federal Rural de Pernambuco (UFRPE). E-mail: <zickelbr@ yahoo.com>.

${ }^{4}$ Programa de Pós-Graduação em Botânica da UFRPE E-mail: <ebaj25 @ yahoo.com.br>.
} 


\section{INTRODUÇÃO}

A vegetação litorânea apresenta uma diversidade fisionômica expressando uma composição que geralmente mescla espécies próprias do litoral com outras provenientes de ecossistemas como floresta atlântica, cerrado e tabuleiros arenosos (FREIRE, 1990; SCARANO, 2002).

Historicamente, no entanto os ecossistemas costeiros no Brasil foram os mais impactados pela ocupação e pelo extrativismo. Tais fatores podem ter levado diversas comunidades vegetais a se extinguir em diversos trechos da costa, apesar de a legislação vigente protegê-las. A região litorânea constitui uma das áreas mais exploradas para o turismo e lazer (ARAUJO e HENRIQUES, 1984).

O Estado de Pernambuco possui 187 km de extensão de área litorânea, onde predominam cotas baixas representadas principalmente por praias arenosas de largura variável, estuários com extensos manguezais, pontais rochosos e restingas (ANDRADE-LIMA, 1960), com as restingas correspondendo, nos dias atuais, a cerca de 5\% do litoral do Estado (ZICKEL et al., 2004).

Do ponto de vista da biodiversidade, pode-se considerar que a zona costeira acolhe quadros naturais, particulares de alta riqueza e relevância ecológica, que os qualifica como importantes fontes de recursos para o ambiente (IBGE, 1996). Apesar da importância ecológica das restingas, o Nordeste possui apenas informações iniciais desse ecossistema quando comparado com os estudos da Região Sudeste, onde já foram listadas cerca de 1.300 espécies (PEREIRA e ARAUJO, 2000).

Para uma melhor compreensão das áreas de restingas são necessárias descrições sobre a vegetação que se pode dar, basicamente, por meio de métodos florísticos e fisionômicos ou estruturais (KENT e COKER, 1992). Dessa forma, a listagem da flora e a caracterização fisionômica contribuem para designar e classificar as vegetações litorâneas, além de diferenciar suas fisionomias (SILVA e BRITEZ, 2005), fornecendo mais conhecimento sobre as restingas do litoral pernambucano.

Atualmente, a vegetação ocorrente nas planícies costeiras arenosas das Regiões Sul e Sudeste do Brasil possui vários aspectos relativamente bem conhecidos, devido ao grande incremento de trabalhos produzidos nos últimos anos (SILVAe BRITEZ, 2005). Dessa forma, o estudo florístico de um remanescente da vegetação de restinga no Estado de Pernambuco é importante, uma vez que existem apenas observações preliminares a respeito dessa vegetação (ANDRADE-LIMA, 1954, 1979). Assim, o objetivo deste estudo foi listar a flora e caracterizar a fisionomia da restinga da praia do Paiva.

\section{MATERIAL E MÉTODOS}

\subsection{Localização da área}

A praia do Paiva situa-se no Município do Cabo de Santo Agostinho, ao sul do Recife, sob as coordenadas $08^{\circ} 07^{\prime} 30^{\prime}$ 'S e $35^{\circ} 00^{\prime} 55^{\prime}$ 'W. Limita-se ao norte com a zona urbanizada da região metropolitana do Recife e o Município de Jaboatão dos Guararapes. Abrange um total de 553 ha, e a área de estudo compreende 147 há, recebendo influência do estuário da Barra de Jangada, formada pelos rios Jaboatão e Pirapama, fazendo parte do Complexo Industrial Portuário de Suape, Pernambuco. Segundo Medeiros (1996), as feições geomorfológicas que melhor representam a área estudada são os terraços marinhos superiores de origem pleistocênica. O solo foi classificado como Neossolo Quartzarênico, de acordo com a classificação da Embrapa (1999).

O litoral sul de Pernambuco possui clima do tipo As', tropical chuvoso, com verão seco, de acordo com a classificação de Köppen. Apresenta variação entre a estação mais seca (outubro-fevereiro), sendo janeiro e fevereiro os meses mais quentes, e outra mais chuvosa (maio-julho), sendo julho o mês mais frio e chuvoso (JACOMINE et al., 1973), com uma precipitação pluviométrica anual em torno de 2.000 mm (MEDEIROS, 1996).

\subsection{Florística}

As coletas foram realizadas na Área de Preservação Ambiental - Reserva Biológica de Mangues e Restingas, em áreas previamente escolhidas com menor interferência antrópica, durante o período de fevereiro de 1997 a maio de 1999; as visitas eram quinzenais, abrangendo a flora fanerogâmica e pteridofítica, em todos os estratos, considerando apenas indivíduos em estádio reprodutivo, sendo excluídos os cipós e as trepadeiras.

O material foi identificado com o auxílio de literatura especializada, e os exemplares não identificados foram enviados para especialistas. Após a identificação, o material foi incorporado ao acervo do Herbário Professor Vasconcelos Sobrinho (PEUFR). A listagem das espécies 
seguiram o que preconizou o sistema de classificação de Cronquist (1981), e as abreviaturas de autores seguiu o Mobot (2007). As formas biológicas seguiram a classificação proposta por Whittaker (1975).

\subsection{Tipos fisionômicos}

Para a denominação dos diferentes tipos fisionômicos ocorrentes na restinga da praia do Paiva, foi utilizada a classificação adaptada de Carvalho (1995) por Silva e Britez (2005). Essa proposta é flexível e utilizável nas diversas áreas litorâneas brasileiras e considera floresta como um tipo vegetacional com predomínio de indivíduos arbóreos, que se podem apresentar organizados em estratos mais ou menos definidos e associados com diferentes formas de vida. E o fruticeto é definido pela presença de espécies arbustivas, eventualmente, com elementos arbóreos isolados que não chegam a formar um estrato contínuo.

\section{RESULTADOS E DISCUSSÃO}

\subsection{Flora}

Foram coletados 571 indivíduos em estádio reprodutivo, perfazendo 124 espécies, distribuídas em 103 gêneros e 55 famílias (Tabela 1).

Tabela 1 - Lista das espécies da restinga da praia do Paiva, Cabo de Santo Agostinho, Pernambuco

Table 1 - List of the species found in the sandy coastal plains Paiva's Beach, Cabo de Santo Agostinho, PernambucoBrazil

\begin{tabular}{|c|c|c|c|}
\hline Família/ Espécie & Hábito & Floresta & Fruticeto \\
\hline \multicolumn{4}{|l|}{ Amaryllidaceae } \\
\hline Hippeastrum stylosum Herb. & Erva & $\mathrm{x}$ & \\
\hline \multicolumn{4}{|l|}{ Anacardiaceae } \\
\hline Anacardium occidentale L. & Árvore & $\mathrm{x}$ & $\mathrm{x}$ \\
\hline Tapirira guianensis Aubl. & Árvore & $\mathrm{x}$ & $\mathrm{x}$ \\
\hline \multicolumn{4}{|l|}{ Annonaceae } \\
\hline Rollinia pickelii Diels & Arbusto & $\mathrm{x}$ & \\
\hline Xylopia laevigata (Mart.) R. E. Fr. & Arbusto & $\mathrm{x}$ & \\
\hline \multicolumn{4}{|l|}{ Araceae } \\
\hline Anthurium affine Schott & Erva & $\mathrm{x}$ & $\mathrm{x}$ \\
\hline Philodendron imbe Schott & Erva & $\mathrm{x}$ & $\mathrm{x}$ \\
\hline \multicolumn{4}{|l|}{ Arecaceae } \\
\hline Acrocomia aculeata (Jacq.) Lodd. ex Mart. & Árvore & $\mathrm{x}$ & \\
\hline Bactris humilis (Wallace) Burret. & Arbusto & $\mathrm{x}$ & \\
\hline Elaeis guineensis Jacq. & Árvore & $\mathrm{x}$ & $\mathrm{x}$ \\
\hline \multicolumn{4}{|l|}{ Asteraceae } \\
\hline Aspilia martii Baker & Erva & & $\mathrm{x}$ \\
\hline Emilia sonchifolia (L.) DC. & Erva & & $\mathrm{x}$ \\
\hline Mikania obovata DC. & Erva & & $\mathrm{x}$ \\
\hline Rolandra argentea Rottb. & Subarbusto & $\mathrm{x}$ & \\
\hline Wedelia trilobata (L.) Hitchc. & Erva & & $\mathrm{x}$ \\
\hline \multicolumn{4}{|l|}{ Aquifoliaceae } \\
\hline Ilex sp. & Arbusto & & $\mathrm{x}$ \\
\hline \multicolumn{4}{|l|}{ Blechnaceae } \\
\hline Blechnum serrulatum Rich. & Erva & $\mathrm{x}$ & \\
\hline \multicolumn{4}{|l|}{ Boraginaceae } \\
\hline Cordia superba Cham. & Arbusto & $\mathrm{x}$ & $\mathrm{x}$ \\
\hline $\begin{array}{l}\text { Tournefortia candidula (Miers) I. M. Johnst. } \\
\text { Bromeliaceae }\end{array}$ & Arbusto escandente & & $\mathrm{x}$ \\
\hline Aechmea muricata (Arruda da Câmara) L.B.Sm. & Erva & & $\mathrm{x}$ \\
\hline \multicolumn{4}{|l|}{ Burseraceae } \\
\hline Protium heptaphyllum (Aubl.) Marchand & Árvore & $\mathrm{x}$ & \\
\hline \multicolumn{4}{|l|}{ Cactaceae } \\
\hline Cereus fernambucensis Lem. & Arbusto & & $-\frac{x}{x}$ \\
\hline
\end{tabular}

R. Árvore, Viçosa-MG, v.31, n.6, p.1121-1130, 2007 
Tabela 1 - Cont.

Table 1 - Cont.

Família/ Espécie

Hábito

Floresta

Fruticeto

Caesalpiniaceae

Chamaecrista ensiformis (Vell.) H.S. Irwin \& Barneby

Chamaecrista ramosa (Vogel) H.S. Irwin \& Barneby

Senna alata (L.) Roxb.

Árvore

Subarbusto

Arbusto

Capparaceae

Capparis flexuosa (L.) L.

Arbusto

Celastraceae

Maytenus distichophylla Mart.

Chrysobalanaceae

Hirtella racemosa Lam.

Clusiaceae

Vismia guianensis (Aubl.) Pers.

Combretaceae

Buchenavia capitata (Vahl) Eichler

Conocarpus erectus L.

Commelinaceae

Commelina obliqua Vahl

Cyperaceae

Abildgaardia scirpoides Nees

Cyperus aggregatus (Willd.) Endl.

Cyperus diffusus Vahl

Cyperus ligularis L.

Cyperus meyenianus Kunth

Cyperus surinamensis Rottb.

Fuirena umbellata Rottb.

Pycreus polystachyos (Rottb.) P. Beauv.

Rhynchospora riparia (Nees) Boeck.

$\begin{array}{lll}\text { Arbusto } & & \mathrm{x} \\ \text { Arbusto } & \mathrm{x} & \mathrm{x} \\ \text { Arbusto } & \mathrm{x} & \mathrm{x} \\ \text { Arbusto } & \mathrm{x} & \\ & & \mathrm{x} \\ \text { Árvore } & \mathrm{x} & \mathrm{x} \\ \text { Arbusto } & & \mathrm{x} \\ & & \\ \text { Erva } & \mathrm{x} & \mathrm{x} \\ & & \mathrm{x} \\ \text { Erva } & & \mathrm{x} \\ \text { Erva } & \mathrm{x} & \mathrm{x} \\ \text { Erva } & \mathrm{x} & \mathrm{x} \\ \text { Erva } & & \mathrm{x} \\ \text { Erva } & & \mathrm{x} \\ \text { Erva } & & \mathrm{x} \\ \text { Erva } & & \\ \text { Erva } & & \\ \text { Erva } & & \end{array}$

\section{Dilleniaceae}

Curatella americana $\mathrm{L}$

Árvore

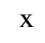

Tetracera breyniana Schltdl.

Arbusto

Eriocaulaceae

Eriocaulon palustre Salzm. ex Steud.

Paepalanthus bifidus (Schrader) Kunth

Paepalanthus tortilis (Bong.) Koern. in C. Martius

Erythroxylaceae

Erythroxylum columbinum Mart.

Erythroxylum passerinum Mart.

Erythroxylum vacciniifolium Mart.

\section{Euphorbiaceae}

Chamaesyce hyssopifolia (L.) Small

Cnidoscolus urens (L.) Arthur

Croton klotzschii (Didr.) Baill.

Croton lobatus L.

Croton sellowii Baill.

Mycrostachys corniculata (Vahl.) Griseb.

Pera glabrata (Schott) Poepp. ex Baill.

Fabaceae

Andira fraxinifolia Benth.

Andira nitida Mart. ex Benth.

Clitoria laurifolia Poir.

Erva

Erva

Erva

Árvore

Arbusto

Arbusto

Subarbusto

Subarbusto

Erva

Subarbusto

Subarbusto

Subarbusto

Árvore

Crotalaria sp.

Árvore

Árvore

Subarbusto

Subarbusto

$\mathrm{X}$

$\mathrm{X}$

$\mathrm{x}$

$\mathrm{x}$

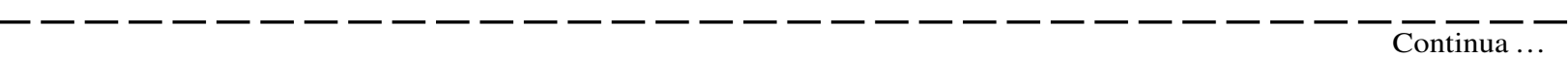

Continued...

R. Árvore, Viçosa-MG, v.31, n.6, p.1121-1130, 2007 
Tabela 1 - Cont.

Table 1 -Cont.

Família/ Espécie

Hábito

Floresta

Fruticeto

Fabaceae

Desmodium barbatum (L.) Benth.

Macroptilium gracile (Poepp. ex. Benth.) Urb.

Stylosanthes guianensis (Aubl.) Sw.

Stylosanthes viscosa (L.) Sw.

Flacourtiaceae

Casearia javitensis Kunth.

Gentianaceae

Schultesia guianensis (Aubl.) Malme

Heliconiaceae

Heliconia angustifolia Hook.

Humiriaceae

Sacoglottis mattogrossensis Malme

Iridaceae

Iris pseudacorus $\mathrm{L}$.

Lamiaceae

Hyptis suaveolens (L.) Poit.

Lauraceae

Ocotea glomerata (Ness.) Mez

Lythraceae

Cuphea flava Spreng.

Malpighiaceae

Byrsonima sericea DC.

Byrsonima gardneriana A. Juss.

Stigmaphyllon paralias A. Juss.

Subarbusto

Subarbusto

Subarbusto

Subarbusto

Arbusto

Erva

Erva

Árvore

Erva

Erva

Árvore

$\mathrm{x}$

Erva

$\mathrm{x}$

Árvore

Arbusto

Subarbusto

$\mathrm{X}$

Malvaceae

Sida ciliaris $\mathrm{L}$.

Melastomataceae

Pterolepis polygonoides (DC.) Triana

Mimosaceae

Abarema cochliacarpos (Gomes) Barneby \& J.W. Grimes

Inga capitata Desv.

Mimosa somnians Humb. \& Bonpl. ex Willd.

Molluginaceae

Mollugo verticillata $\mathrm{L}$.

Myrtaceae

Calyptranthes dardanoi Mattos

Eugenia ferreiraeana O. Berg

Eugenia punicifolia (Kunth) DC.

Marlierea aff. schottii (O. Berg) D. Legrand

Marlierea strigipes $\mathrm{O}$. Berg

Myrcia hirtiflora DC.

Myrcia aff. laroutteana Cambess.

Myrcia multipla D. Legrand

Myrcia rotundifolia (O. Berg) Kiaersk

Myrcia sylvatica (G. Mey.) DC.

Psidium guineense Sw.

Erva

$\mathrm{x}$

Nyctaginaceae

Guapira pernambucensis (Casar.) Lundell.

Erva

$\mathrm{X}$

Erva

Árvore $\quad x$

Árvore

Subarbusto

$\mathrm{X}$

Erva

$\mathrm{X}$

Arbusto

Arbusto

Arbusto

Arbusto

Arbusto

Arbusto

Arbusto

Arbusto

Arbusto

Arbusto

Arbusto

$\mathrm{x}$

$\mathrm{x}$

$x$

Ochnaceae

Ouratea crassa Tiegh.

$\begin{array}{lll}\text { Arbusto } & \text { X } & \text { X }\end{array}$

Arbusto

$-\frac{x}{\text { Continua ... }}-\frac{\mathrm{x}}{\mathrm{C}}$

Continued...

R. Árvore, Viçosa-MG, v.31, n.6, p.1121-1130, 2007 
Tabela 1 - Cont.

Table 1 - Cont.

\begin{tabular}{|c|c|c|c|}
\hline Família/ Espécie & Hábito & Floresta & Fruticeto \\
\hline \multicolumn{4}{|l|}{ Orchidaceae } \\
\hline Cyrtopodium intermedium Brade & Erva & $\mathrm{x}$ & $\mathrm{x}$ \\
\hline Habenaria petalodes Lindl. & Erva & & $\mathrm{x}$ \\
\hline Oeceoclades maculata (Lindl.) Lindl. & Erva & $\mathrm{x}$ & \\
\hline Prescottia stachyodes (Sw.) Lindl. & Erva & & $\mathrm{x}$ \\
\hline \multicolumn{4}{|l|}{ Poaceae } \\
\hline Eleusine indica (L.) Gaertn. & Erva & & $\mathrm{x}$ \\
\hline Eragrostis ciliaris (L.) R. Br. & Erva & & $\mathrm{x}$ \\
\hline Eragrostis rufescens Schrad. ex Schult. & Erva & & $\mathrm{x}$ \\
\hline Paspalum maritimum Trin. & Erva & & $\mathrm{x}$ \\
\hline \multicolumn{4}{|l|}{ Polygalaceae } \\
\hline Polygala violacea Aubl. & Erva & & $\mathrm{x}$ \\
\hline \multicolumn{4}{|l|}{ Polygonaceae } \\
\hline Coccoloba laevis Casar. & Arbusto & $\mathrm{x}$ & $\mathrm{x}$ \\
\hline \multicolumn{4}{|l|}{ Polypodiaceae } \\
\hline Polypodium decumanum Willd. & Erva & & $\mathrm{x}$ \\
\hline \multicolumn{4}{|l|}{ Rubiaceae } \\
\hline Borreria scabiosoides Cham. \& Schltdl. & Erva & & $\mathrm{x}$ \\
\hline Borreria verticillata (L.) G. Мey. & Erva & $\mathrm{x}$ & $\mathrm{x}$ \\
\hline Guettarda platypoda DC. & Arbusto & $\mathrm{x}$ & $\mathrm{x}$ \\
\hline Mitracarpus frigidus (Willd. ex Roem. \& Schult.) K. Schum. & Erva & $\mathrm{x}$ & $\mathrm{x}$ \\
\hline Richardia grandiflora (Cham. \& Schltdl.) Steud. & Erva & & $\mathrm{x}$ \\
\hline Tocoyena brasiliensis Mart. & Arbusto & & $\mathrm{x}$ \\
\hline \multicolumn{4}{|l|}{ Sapotaceae } \\
\hline Manilkara salzmannii (A. DC.) H.J. Lam. & Árvore & $\mathrm{x}$ & $\mathrm{x}$ \\
\hline \multicolumn{4}{|l|}{ Simaroubaceae } \\
\hline Simaba cuneata A. St.-Hil. \& Tul. & Árvore & $\mathrm{x}$ & $\mathrm{x}$ \\
\hline \multicolumn{4}{|l|}{ Scrophulariaceae } \\
\hline Scoparia dulcis L. & Erva & $\mathrm{x}$ & $\mathrm{x}$ \\
\hline \multicolumn{4}{|l|}{ Solanaceae } \\
\hline Cestrum parqui L'Hér. & Erva & & $\mathrm{x}$ \\
\hline Schwenckia americana $\mathrm{L}$. & Erva & $\mathrm{x}$ & \\
\hline Solanum americanum Mill. & Arbusto & & $\mathrm{x}$ \\
\hline Solanum paludosum Moric. & Arbusto & $\mathrm{x}$ & $\mathrm{x}$ \\
\hline \multicolumn{4}{|l|}{ Sterculiaceae } \\
\hline Waltheria indica $\mathrm{L}$. & Subarbusto & & $\mathrm{x}$ \\
\hline \multicolumn{4}{|l|}{ Verbenaceae } \\
\hline Lantana camara $\mathrm{L}$. & Subarbusto & & $\mathrm{x}$ \\
\hline Lippia sp. & Erva & & $\mathrm{x}$ \\
\hline Stachytarpheta cayennensis (Rich.) Vahl. & Subarbusto & $\mathrm{x}$ & $\mathrm{x}$ \\
\hline \multicolumn{4}{|l|}{ Vochysiaceae } \\
\hline Qualea cryptantha (Spreng.) Warm. & Árvore & $\mathrm{x}$ & \\
\hline \multicolumn{4}{|l|}{ Xyridaceae } \\
\hline Xyris jupicai Rich. & Erva & & $\mathrm{x}$ \\
\hline
\end{tabular}

As famílias com maior número de espécies na restinga da praia do Paiva foram Myrtaceae (11 espécies), Cyperaceae (10), Fabaceae (8), Euphorbiaceae (7), Rubiaceae (6), Asteraceae (5), Orchidaceae, Solanaceae e Poaceae (4) e Malpighiaceae, Arecaceae, Eriocaulaceae, Erythroxylaceae, Caesalpiniaceae, Mimosaceae e Verbenaceae (3). Trinta e três famílias apresentaram uma espécie, correspondendo a $60 \%$ do total de famílias.
A riqueza da família Myrtaceae tem sido apontada como uma característica da floresta atlântica, como relatado com relação a outras áreas de florestas de restinga e tabuleiros arenosos no Nordeste, Norte e Sudeste do país (PEIXOTO e GENTRY, 1990; ASSUMPÇÃO NASCIMENTO, 2000; CARVALHO et al., 2000; OLIVEIRA-FILHO e FONTES, 2000; ASSIS et al., 2004; SILVA e BRITEZ, 2005). A ocorrência de

R. Árvore, Viçosa-MG, v.31, n.6, p.1121-1130, 2007 
Myrtaceae na área é representativa, e as espécies dessa família mais visualizadas foram Myrcia multipla, Eugenia punicifolia, Marlierea schottii, Myrcia hirtiflora e Myrcia rotundifolia. Portanto, a representatividade desta família sugere afinidade com as áreas de floresta atlântica, como foi indicado por Freire (1990).

A família Cyperaceae foi bem expressiva, com maior número de indivíduos na formação fruticeto, provavelmente pelo fato de a própria fisionomia favorecer esse desenvolvimento, devido à facilidade dessas espécies em colonizar as margens e estar associadas a espécies pioneiras (CABRAL-FREIRE e MONTEIRO, 1993), predominantemente hemicriptófitas, mantendo representantes no banco de semente, devido à proteção da gema (MEIRA-NETO et al., 2005).

A família Fabaceae apresentou várias espécies, sendo a maioria conhecida como fixadora de areia (CABRAL-FREIRE e MONTEIRO, 1993), que propiciam melhores condições edáficas (ARAÚJO et al., 2006). As espécies mais comuns tanto na área de estudo quanto em outras áreas litorâneas foram Stylosanthes guianensis, Stylosanthes viscosa, Crotalaria sp, Clitoria laurifolia e Desmodium barbatum (SILVA e OLIVEIRA, 1989; OLIVEIRA-FILHO CARVALHO, 1993; PEREIRA e ASSIS, 2000). Euphorbiaceae também teve destaque na restinga do Paiva, e em outras áreas fitogeográficas (ANDRADE-LIMA, 1960; BORÉMeOLIVEIRA-FILHO, 2002), como exemplo de espécies dessa família temse Croton sellowii, Croton lobatus e Croton klotzschii, consideradas como pioneiras, contribuindo, assim, para a melhoria do solo (RICKLEFS, 2003).

As espécies lenhosas mais comuns foram Anacardium occidentale, Tapirira guianensis, Protium heptaphyllum, Byrsonima sericea, Myrcia rotundifolia e Marlierea schotti. Estas espécies são encontradas na maioria das restingas do Nordeste, no entanto, ainda não foi possível detectar espécies endêmicas, talvez, devido à incipiência dos estudos na Região Nordeste (ZICKEL et al., 2004) ou, mesmo, à proximidade de outros ecossistemas com a restinga, não havendo tempo suficiente para especiação (SCARANO, 2002).

As fisionomias observadas na praia do Paiva foram: a floresta não inundável e o fruticeto aberto não inundável.

A floresta não inundável foi caracterizada por três estratos. O estrato superior, composto por árvores variando de 6 a $15 \mathrm{~m}$ de altura, podendo ocorrer indivíduos emergentes com cerca de $20 \mathrm{~m}$ de altura e copas justapostas. As espécies arbóreas que caracterizaram o estrato superior foram: Protium heptaphyllum, Buchenavia capitata, Inga capitata, Qualea cryptantha e Simaba cuneata. As duas primeiras espécies também se destacaram no dossel das restingas estudadas no Sudeste por Assumpção e Nascimento (2000) e Assis et al. (2004). O estrato intermediário (sub-bosque) é formado por arvoretas e arbustos de troncos finos, com altura de 2 a $8 \mathrm{~m}$, com indivíduos menores e esparsos, em que Rollinia pickelli, Casearia javitensis, Stachytarpheta cayennensis, Stylosanthes guianensis e Croton lobatus foram mais expressivas.

O estrato inferior foi constituído basicamente por ervas, com predomínio de espécies rizomatosas, reptantes e eretas, proporcionando uma cobertura descontínua. Dentre as herbáceas do estrato inferior, é comum encontrar Habenaria petalodes, Prescottia stachyoides, Schultesia guianensis, Hippeastrum stylosum e Iris pseudacorus, desenvolvendo-se em pontos sombreados e sobre uma camada de serrapilheira.

Foram encontradas duas famílias de pteridófitas (Blechnaceae e Polypodiaceae) no estrato inferior. A fisionomia dessa formação é marcada pela cobertura do solo por indivíduos de Blechnum serrulatum, que delimita a área de transição para o mangue e para a descida em direção ao rio, enquanto Polypodium decumanum se encontra no interior da floresta, sobre a serrapilheira e próximo ao caule das árvores. Blechnum serrulatum foi expressiva juntamente com outras espécies de pteridófitas no estrato inferior da fisionomia floresta da ilha do Mel, Paraná (SILVA e BRITEZ, 2005), sendo um componente importante nessa feição fisionômica.

No fruticeto aberto não inundável, foram encontradas predominantemente espécies arbustivas, associadas às herbáceas, eventualmente com elementos arbóreos isolados que não formavam um estrato contínuo, além de não ocorrer inundação na área. Essa fisionomia possui o hábito arbustivo com $24,8 \%$ nesse componente, com uma faixa de variação de 2 a $5 \mathrm{~m}$ de altura e alguns indivíduos arbóreos isolados chegando a atingir 15 $\mathrm{m}$ de altura. O estrato herbáceo é constituído pelas famílias Poaceae, Cyperaceae, Xyridaceae, Bromeliaceae, Cactaceae e Eriocaulaceae, cobrindo grande extensão do solo. Esse fato também foi observado por MeiraNeto et al. (2005) no litoral sul da Bahia, onde as espécies herbáceas se destacaram, contribuindo com um porte

R. Árvore, Viçosa-MG, v.31, n.6, p.1121-1130, 2007 
mais baixo para a fisionomia arbustiva. Apresenta agrupamentos arbustivos (moitas) que se distribuem intercalados com áreas desprovidas de vegetação. Essas moitas, além do porte dos indivíduos, também foram observadas na restinga da ilha do Mel, PR, compondo a fisionomia fruticeto (SILVAe BRITEZ, 2005), tornando essa característica expressiva para essa formação fisionômica.

Nas áreas abertas, entre as moitas, foi observada uma cobertura vegetal heterogênea constituída por espécies herbáceas e subarbustivas como Stigmaphyllon paralias, Stylosanthes guianensis, Xyris jupicai, Cuphea flava e Chamaecrista ramosa. Tais espécies, juntamente com Staelia galioides, Sauvagesia tenella e Stemodia foliosa, também foram registradas nas áreas desnudas da restinga de Guadalupe (CANTARELLI, 2003).

As espécies de Araceae foram encontradas, na maioria das vezes, sob as moitas de Myrtaceae ou de Anacardium occidentale, cujas copas alcançam o chão e propiciam o desenvolvimento de Anthurium affine e Philodendron imbe. Cereus fernambucensis (Cactaceae) foi observada tanto isolada quanto agrupada, formando extensa população sobre um solo arenoso e desnudo. Essa mesma espécie tem papel de destaque na restinga de Barra de Maricá, Rio de Janeiro, estudada por Ribas (1992 apud ZALUAR e SCARANO, 2000), devido à sua importância no processo de agrupamento de outras espécies, colonizando áreas com pouca vegetação.

Das 124 espécies encontradas, 48 são comuns às duas fisionomias. Dezessete espécies foram exclusivas da fisionomia floresta, entre as quais Curatella americana, Inga capitata e Qualea cryptantha, que se destacaram entre os indivíduos arbóreos devido à copa e ao porte. No fruticeto, 58 espécies foram exclusivas, mostrando maior riqueza de espécies nessa fisionomia, com $29 \%$ de herbáceas e $12 \%$ de arbustivas, em que as mais visualizadas foram Stylosanthes viscosa, Eragrostis ciliaris, Cereus fernambucensis, Senna alata e Casearia javitensis. O grande número de espécies herbáceas encontradas nos diferentes estudos do litoral de Pernambuco (CANTARELLI, 2003; LIRA, 2004) justifica-se por apresentarem famílias intolerantes à sombra, pela diferenciação quanto ao tipo reprodutivo (CABRAL-FREIRE e MONTEIRO, 1993) e a grande quantidade de representantes no banco de sementes (BOND e van WILGEN, 1996).

R. Árvore, Viçosa-MG, v.31, n.6, p.1121-1130, 2007
As restingas de Sirinhaém e Paiva, litoral sul de Pernambuco, são semelhantes em relação às espécies, diferindo, contudo, no arranjo fisionômico. Essa diferença se deve, provavelmente, aos diferentes níveis de antropização (ZICKEL et al., 2004) ou essa semelhança seria influenciada pelos ecossistemas adjacentes, nesse caso a floresta atlântica.

Dessa forma, a conservação das áreas adjacentes à floresta atlântica, entre elas as restingas, é importante por também apresentar alta diversidade (SCARANO, 2002; ASSIS et al., 2004). Contudo, para a escolha da área deve ser considerada a dimensão, o histórico e o uso do fragmento, para assim obter maior eficiência em manter populações viáveis de plantas e os seus processos ecológicos (SCARIOT et al., 2003). Diante da riqueza florística e de seus aspectos fisionômicos, a restinga do Paiva apresenta potencial ecológico que justifica a maior proteção desse ecossistema perante as ações antrópicas, servindo como referência para outras áreas de restinga carentes de proteção.

\section{AGRADECIMENTOS}

Ao CNPq, pela concessão da bolsa de estudo ao primeiro autor, ao Prof. Jorge Tamashiro, que auxiliou, em parte, as identificações e a leitura inicial do manuscrito e ao Prof. Dr. Sandro Menezes, pelas críticas construtivas ao manuscrito.

\section{REFERÊNCIAS}

ANDRADE-LIMA, D. Primeira contribuição para o conhecimento da flora do Cabo de Santo Agostinho. In: CONGRESSO NACIONAL DA SOCIEDADE BOTÂNICA DO BRASIL, 4., 1954, Recife. Anais... Recife: 1954. p.48-57.

\section{ANDRADE-LIMA, D. Estudos}

fitogeográficos de Pernambuco. Recife: Instituto de Pesquisa Agronômica de Pernambuco, 1960. (Publicação, 2).

ANDRADE-LIMA, D. A flora e a vegetação da área Janga-Mamanguape/Paulista - Pernambuco. In: CONGRESSO NACIONAL DE BOTÂNICA, 30., 1979, Campo Grande. Anais... Campo Grande, 1979. p.179-190.

ARAUJO, D. S. D.; HENRIQUES, R. P. B. Análise florística das restingas do estado do Rio de Janeiro. In: LACERDA, L.D. et al. (Ed.).

Restingas: origem, estrutura e processos. Niterói: CEUFF, 1984. p.47-60. 
ARAÚJO, F. S. et al. Estrutura da vegetação arbustivo-arbórea colonizadora de uma área degradada por mineração de caulim, Brás Pires, MG. Revista Árvore, v.30, n.1, p.07-116, 2006.

ASSIS, A. M.; THOMAZ, L. D.; PEREIRA, O. J. Florística de um trecho de floresta de restinga no município de Guarapari, Espírito Santo, Brasil. Acta Botanica Brasilica, v.18, n.1, p.191-201, 2004.

ASSUMPÇÃO, J.; NASCIMENTO, M. T. Estrutura e composição florística de quatro formações vegetais de restinga no complexo lagunar Grussaí/ Iquipari, São João da Barra, RJ, Brasil. Acta Botanica Brasilica, v.14, n.3, p.301-315, 2000.

BOND, W. J.; van WILGEN, B. W. Fire and plants. London: Chapman \& Hall, 1996. 263p.(Population and community biology, 14).

BORÉM, R. A. T.; OLIVEIRA-FILHO, A. T. Fitossociologia do estrato arbóreo em uma topossequiência alterada de Mata Atlântica, no Município de Silva Jardim-RJ, Brasil. Revista Árvore, v.26, n.6, p.727-742, 2002.

CABRAL-FREIRE, M. C.; MONTEIRO, R. Florística das praias da Ilha de São Luiz, estado do Maranhão (Brasil): Diversidade de espécies e sua ocorrências no litoral brasileiro. Acta Amazônica, v.23, n.2-3, p.125-140, 1993.

CANTARELli, J. R. R. Florística e estrutura de uma restinga da Área de Proteção Ambiental (APA) de Guadalupe - litoral sul de

Pernambuco. 2003. 75f. Dissertação (Mestrado em Botânica) - Universidade Federal Rural de Pernambuco, Recife, 2003.

CARvalho, V. C. Proposta de um sistema flexível de classificação fitogeográfica: Enfase no uso de sensoriamento remoto multiestágio. São José dos Campos: Instituto Nacional de Pesquisas Espaciais - INPE, 1995. 52p.

CARVALHO, D. A. et al. Florística e estrutura da vegetação arbórea de um fragmento de floresta semidecidual às margens do reservatório da usina hidrelétrica Dona Rita, Itambé do Mato Dentro, MG. Acta Botanica Brasilica, v. 14, n.1, p.37-55, 2000.
CRONQUIST, A. The evolution and classification of flowering plants. New York: The New York Botanical Garden, 1981. 555p.

\section{EMPRESABRASILEIRADEPESQUISA}

AGROPECUÁRIA- EMBRAPA. Centro Nacional de Pesquisa de Solos Sistema brasileiro de classificação de solos. Rio de janeiro: 1999. 412p.

FREIRE, M. S. B. Levantamento florístico do Parque Estadual das Dunas de Natal. Acta Botanica Brasilica, v.4, n.2, p.41-59, 1990. (Suplemento).

\section{INSTITUTOBRASILEIRODEGEOGRAFIAE}

ESTATISTICA - IBGE. Macro desenvolvimento da zona costeira. Rio de Janeiro: 1996. 60p.

\section{JACOMINE, P. T. et al. Levantamento} exploratório-reconhecimento de solos do estado de Pernambuco. Recife: SUDENE/Divisão de Pesquisa Pedológica, 1973. v.1. 175p. (Boletim Técnico 26, Pedologia, 14)

\section{KENT, M.; COKER, P. Vegetation} description and analysis - a practical approach. London: Belhaven Press, 1992.

LIRA, S. S. Flora vascular da restinga de Ariquindá, APA de Guadalupe, Tamandaré, Pernambuco. 2004. 57f. Dissertação (Mestrado em Botânica) - Universidade Federal Rural de Pernambuco, Recife, 2004.

\section{MEDEIROS, A. B. Compartimentações geológico-geomorfológica e geoambiental na faixa costeira sul da região metropolitana do Recife - Folha Ponte dos Carvalhos (SC 25-V-A-III/1- SE) e a Folha Santo Agostinho (SC 25- V-A-III/3-NO). 1996. 146f. Dissertação (Mestrado em Geociências) - Universidade Federal de Pernambuco, Recife, 1996.}

MEIRA-NETO, J. A. et al. Composição Florística, Espectro Biológico e Fitofisionomia da vegetação de muçununga nos Municípios de Caravelas e Mucuri, Bahia. Revista Árvore, v.29, n.1, p.139-150, 2005.

MOBOT. Disponível em: (http:// mobot.mobot.org/W3 T/Search/ vast.html). Acessado em janeiro de 2007.

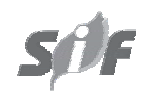

R. Árvore, Viçosa-MG, v.31, n.6, p.1121-1130, 2007 
OLIVEIRA-FILHO, A. T.; CARVALHO, D. A. Florística e fisionomia da vegetação no extremo norte do litoral da Paraíba. Revista Brasileira de Botânica, v.16, n.1, p.115-130, 1993.

OLIVEIRA-FILHO, A. T.; FONTES, M. A. L. Patterns of floristic differentiation among Atlantic forests in southeastern Brazil and the influence of climate. Biotropica, v.32, n.4b, p.793-810, 2000.

PEREIRA, O. J.; ASSIS, A. M. Florística da restinga de Camburi. Acta Botanica Brasilica, v.14, n.1, p.99-111, 2000.

PEIXOTO, A. L.; GENTRY,A. Diversidade e composição florística de mata de tabuleiro na Reserva de Linhares (Espírito Santo, Brasil). Revista Brasileira de Botânica, v.13, p.19-25, 1990.

PEREIRA, O. J.; ARAUJO, D. S. D. Análise florística das restingas dos estados do Espírito Santo e Rio de Janeiro. In: ESTEVES, F. A.; LACERDA, L. D. (Ed.). Ecologia de Restingas e Lagoas Costeiras Macaé: UFRJ/ NUPEM, 2000. p.25-63.

RICKLEFS, R. E. A economia da natureza. 5.ed. Rio de Janeiro: Guanabara Koogan, 2003.

SCARANO, F. R. Structure, function and floristic relantioships of plants communities in stressful habitats marginal to Brazilian Atlantic Rainforest. Annals of Botany, v.90, p.517-524, 2002.
SCARIOT, S. R. et al. Vegetação e flora. In: RAMBALDI, D.M.; OLIVEIRA, D. A. S. (Org.). Fragmentação de ecossistemas: Causas, efeitos sobre a biodiversidade e recomendações de políticas públicas. Brasília: MMA/ SBF, 2003.p.104-123.

SILVA, J. G.; OLIVEIRA, A. S. A. vegetação de restinga no município de Maricá, RJ. Acta Botanica Brasilica, v.3, n.2, p.253-272, 1989. (Suplemento).

SILVA, S. M.; BRITEZ, R. M. A vegetação da Planície Costeira. In: MARQUES, M. C. M.; BRITEZ, R. M. (Org.). História Natural e conservação da Ilha do Mel. Curitiba: Universidade Federal do Paraná, 2005.p.49-84.

WhitTAKER, R. H. Communities and ecossystems. New York: MacMillan, 1975.

ZALUAR, H. L. T.; SCARANO, F. R. Facilitação em restingas de moitas: Um século de buscas por espécies focais. In: ESTEVES, F.A.; LACERDA, L. D. (Ed.). Ecologia de restingas e lagoas costeiras. Rio de Janeiro: NUPEM/UFRJ, 2000. p.3-23.

ZICKEL, C. S. et al. Flora e vegetação das restingas no Nordeste Brasileiro. In: ESKINAZILEÇA, E.; NEUMANN-LEITÃO, S.; COSTA, M. F. (Org.) Oceanografia: um cenário tropical. Recife: Bargaço, 2004. p.689-701. 